THE KURUME MEDICAL JOURNAL

1973 Vol.20, No.4, P.199-209

\title{
EPIDEMIOLOGICAL STUDIES ON TUBERCULOSIS IN KURUME CITY (REPORT П)
}

\author{
AN ANALYSIS OF THE CHIEF FACTORS OF SELF-DISCHARGE \\ OF THE FORCEDLY ADMITTED TUBERCULOUS PATIENTS
}

\author{
HIROJI ESAKI \\ Department of Environmental Health, Kurume University \\ School of Medicine, Kurume, Japan
}

(Received for publication November 10, 1973)

\begin{abstract}
The author performed an analysis of the discharge factors of the tuberculous patients who discharged from hospitals by themselves before getting favorable improvement. The patients who were discharged in favorable condition were chosen as controls. 227 forcedly admitted patients (142 males and 85 females) discharged in the period of 1969 to 1971 were investigated.

The following factors were found to be the main causes of self-discharge.

1) Roentogenological finding- self-discharge occurred more often in patients with worse findings

2) Low level of education

3) Uncooperativeness toward treatment

4) Lack of hands for housekeeping for female patients and

5 ) Uncooperativeness of the patient's family

From the date, we know that to complete a long-term treatment needs not only the public expenditure for the medical fee but also the patient's own awareness of the disease, cooperativeness toward treament and warm attention of the family.
\end{abstract}

The author, as a consultant, has been taking part in the management of the tuberculous patients at Kurume Health Center since 1961. An analysis of the causes of death of the registered tuberculous patients ${ }^{11}$ and the yearly transition of tuberculosis ${ }^{2)}$ have been reported. And the prevalence, the incidence and the state of treatment of tuberculosis of Fukuoka Prefecture compared with that of the whole country was also reported in the previous report ${ }^{3)}$.

Though the state of treatment of tuberculosis of Kurume City was better than the average of the whole country, the author noticed that there were many patients who discharged by themselves before getting much improvement. So, in order to get a more complete treatment for the tuberculous patients, the author performed this study. The outline was reported in the Kyushu Local Conference of the Japanese Society for Tuberculosis in November of $1970^{4)}$.

\section{METHOD}

From the tuberculous patients registered at Kurume Health Center, those 
TABLE 1

By age and sex, 1969-1971, Kurume

\begin{tabular}{|c|c|c|c|c|c|c|c|c|c|}
\hline \multirow[b]{2}{*}{$\begin{array}{l}\text { Age } \\
\text { (yr.) }\end{array}$} & \multicolumn{3}{|c|}{ Male } & \multicolumn{3}{|c|}{ Female } & \multicolumn{3}{|c|}{ Total } \\
\hline & $\begin{array}{l}\text { Self- } \\
\text { discharge }\end{array}$ & $\mid \begin{array}{l}\text { Remission } \\
\text { discharge }\end{array}$ & Total & $\begin{array}{l}\text { Self - } \\
\text { discharge }\end{array}$ & $\mid \begin{array}{l}\text { Remission } \\
\text { discharge }\end{array}$ & Total & $\begin{array}{l}\text { Self - } \\
\text { discharge }\end{array}$ & $\begin{array}{r}\text { Remission } \\
\text { discharge }\end{array}$ & Total \\
\hline $0 \sim 9$ & 0 & 0 & 0 & 0 & 0 & 0 & 0 & 0 & 0 \\
\hline $10 \sim 19$ & 1 & 4 & 5 & 1 & 6 & 7 & 2 & 10 & 12 \\
\hline $20 \sim 29$ & 8 & 9 & 17 & 4 & 11 & 15 & 12 & 20 & 32 \\
\hline $30 \sim 39$ & 12 & 22 & 34 & 7 & 10 & 17 & 19 & 32 & 51 \\
\hline $40 \sim 49$ & 13 & 13 & 26 & 4 & 17 & 21 & 17 & 30 & 47 \\
\hline $50 \sim 59$ & 9 & 11 & 20 & 4 & 4 & 8 & 13 & 15 & 28 \\
\hline $60 \sim 69$ & 8 & 15 & 23 & 6 & 6 & 12 & 14 & 21 & 35 \\
\hline $70 \sim$ & 11 & 6 & 17 & 2 & 3 & 5 & 13 & 9 & 22 \\
\hline Total & 62 & 80 & 142 & 28 & 57 & 85 & 90 & 137 & 227 \\
\hline
\end{tabular}

TABLE 2

By period of admission, 1969-1971, Kurume

\begin{tabular}{|c|c|c|c|c|c|c|c|c|c|}
\hline \multirow{2}{*}{$\begin{array}{l}\text { Period of } \\
\text { admission } \\
\quad(\mathrm{yr} . / \mathrm{m} .)\end{array}$} & \multicolumn{3}{|c|}{ Male } & \multicolumn{3}{|c|}{ Female } & \multicolumn{3}{|c|}{ Total } \\
\hline & $\begin{array}{l}\text { Self- } \\
\text { discharge }\end{array}$ & $\begin{array}{l}\text { Remission } \\
\text { discharge }\end{array}$ & Total & $\begin{array}{l}\text { Self- } \\
\text { discharge }\end{array}$ & $\begin{array}{l}\text { Remission } \\
\text { discharge }\end{array}$ & Total & $\begin{array}{l}\text { Self- } \\
\text { discharge }\end{array}$ & $\begin{array}{l}\text { Remission } \\
\text { discharge }\end{array}$ & Total \\
\hline$\sim 0 / 5$ & 8 & 4 & 12 & 3 & 5 & 8 & 11 & 9 & 20 \\
\hline $0 / 6 \sim 0 / 11$ & 11 & 13 & 24 & 3 & 15 & 18 & 14 & 28 & 42 \\
\hline $1 / 0 \sim 1 / 5$ & 7 & 15 & 22 & 4 & 10 & 14 & 11 & 25 & 36 \\
\hline $1 / 6 \sim 1 / 11$ & 7 & 9 & 16 & 2 & 5 & 7 & 9 & 14 & 23 \\
\hline $2 / 0 \sim 2 / 5$ & 6 & 5 & 11 & 2 & 5 & 7 & 8 & 10 & 18 \\
\hline $2 / 6 \sim 2 / 11$ & 6 & 9 & 15 & 4 & 6 & 10 & 10 & 15 & 25 \\
\hline $3 / 0 \sim 3 / 5$ & 2 & 5 & 7 & 3 & 0 & 3 & 5 & 5 & 10 \\
\hline $3 / 6 \sim 3 / 11$ & 2 & 4 & 6 & 2 & 3 & 5 & 4 & 7 & 11 \\
\hline $4 / 0 \sim 4 / 5$ & 1 & 5 & 6 & 2 & 1 & 3 & 3 & 6 & 9 \\
\hline $4 / 6 \sim 4 / 11$ & 2 & 3 & 5 & 0 & 2 & 2 & 2 & 5 & 7 \\
\hline $5 / 0 \sim 5 / 5$ & 3 & 1 & 4 & 1 & 3 & 4 & 4 & 4 & 8 \\
\hline $5 / 6 \sim 5 / 11$ & 0 & 0 & 0 & 1 & 0 & 1 & 1 & 0 & 1 \\
\hline $6 / 0 \sim 6 / 5$ & 1 & 2 & 3 & 1 & 1 & 2 & 2 & 3 & 5 \\
\hline $6 / 6 \sim 6 / 11$ & 2 & 1 & 3 & 0 & 0 & 0 & 2 & 1 & 3 \\
\hline $7 / 0 \sim$ & 2 & 2 & 4 & 0 & 1 & 1 & 2 & 3 & 5 \\
\hline Total & 60 & 78 & 138 & 28 & 57 & 85 & 88 & 135 & 223 \\
\hline Mean & 2.33 & 2.40 & 2.36 & 2. 54 & 2.04 & 2. 19 & 2.38 & 2.24 & 2.29 \\
\hline S.D. & 1. 967 & 1. 706 & 1.807 & 1. 656 & 1. 671 & 1. 661 & 1. 844 & 1.692 & 1. 755 \\
\hline
\end{tabular}


discharged by themselves during the period of 1969 to 1971 and those discharged in favorable condition of the same period were chosen for comparative study. The total number of patients was 227 . The distribution by age and sex was shown in Table 1. Among the 142 male patients, 62 were self-discharged and 80 were remission discharged. And among the 85 female patients, 28 were self-discharged and 57 were remission discharged. With the informations obtained from the interviews of the patients and the public health nurses, records of the Tuberculosis Council of the Center and the registration cards, the author has analysed and compared the period of admission, occupation, way of case discovery, roentogenological finding, bacteriological state on admission, number of changing doctors or hospitals, the level of education, patient's attitude toward treatment, the income level, the duty of household economy, hands for housekeeping and the attitude of the family toward treatment between the 2 groups.

\section{RESULTS}

1) The period of admission (Table 2)

The distribution of period of admission with an interval of 6 months was shown in Table 2. The average admission period of the male patients was 2.33 years for the self-discharged and 2. 40 years for the remission discharged, and that of the female patients was 2.54 years for the self-discharged and 2.04 years for the remission discharged. The average admission period of the female patients discharged in favorable condition was a little shorter, but there was no significant difference statistically.

2) Occupation (Table 3)

The distribution by occupation was shown in Table 3. More patients were merchants and craftsmen followed by regularly employed laborers and farmers. The tendency was thought to be due to that the social insurance was not applicable to most of them. But both male and female patients showed no significant difference in occupation between each group.

3) Way of case discovery (Table 4)

The way of case discovery was classified into physical check-up, mass survey and on receiving treatment at medical facilities. Of the male patients discharged by themselves, $1.6 \%$ were discovered by physical check-up, $24.2 \%$ by mass survey and $74.2 \%$ on receiving treatment at medical facilities. None of the male patients discharged in favorable condition was found by physical check-up, $8.8 \%$ were discovered by mass survey and $91.2 \%$ on receiving treatment at medical facilities. Those discharged by themselves were discovered more than those discharged in favorable condition by way of mass survey with significant difference. There was no significant difference in way of discovery for female patients.

4) Roentogenological finding on admission (Table 5)

The roentogenological finding was classified according to the classification presented by the Japanese Society for Tuberculosis. $11.3 \%$ of the self-discharged belong to Type I, $79.0 \%$ to Type II and $9.7 \%$ to Type III. Among those discharged in favorable condition, $2.5 \%$ were Type I, $73.7 \%$ were Type II and $23.8 \%$ were Type III. The selfdischarged were predominant to Type I and Type II and less to Type III $(p<0.02)$. There was no significant difference between female patients. 
TABLE 3

By occupation, 1961-1971, Kurume

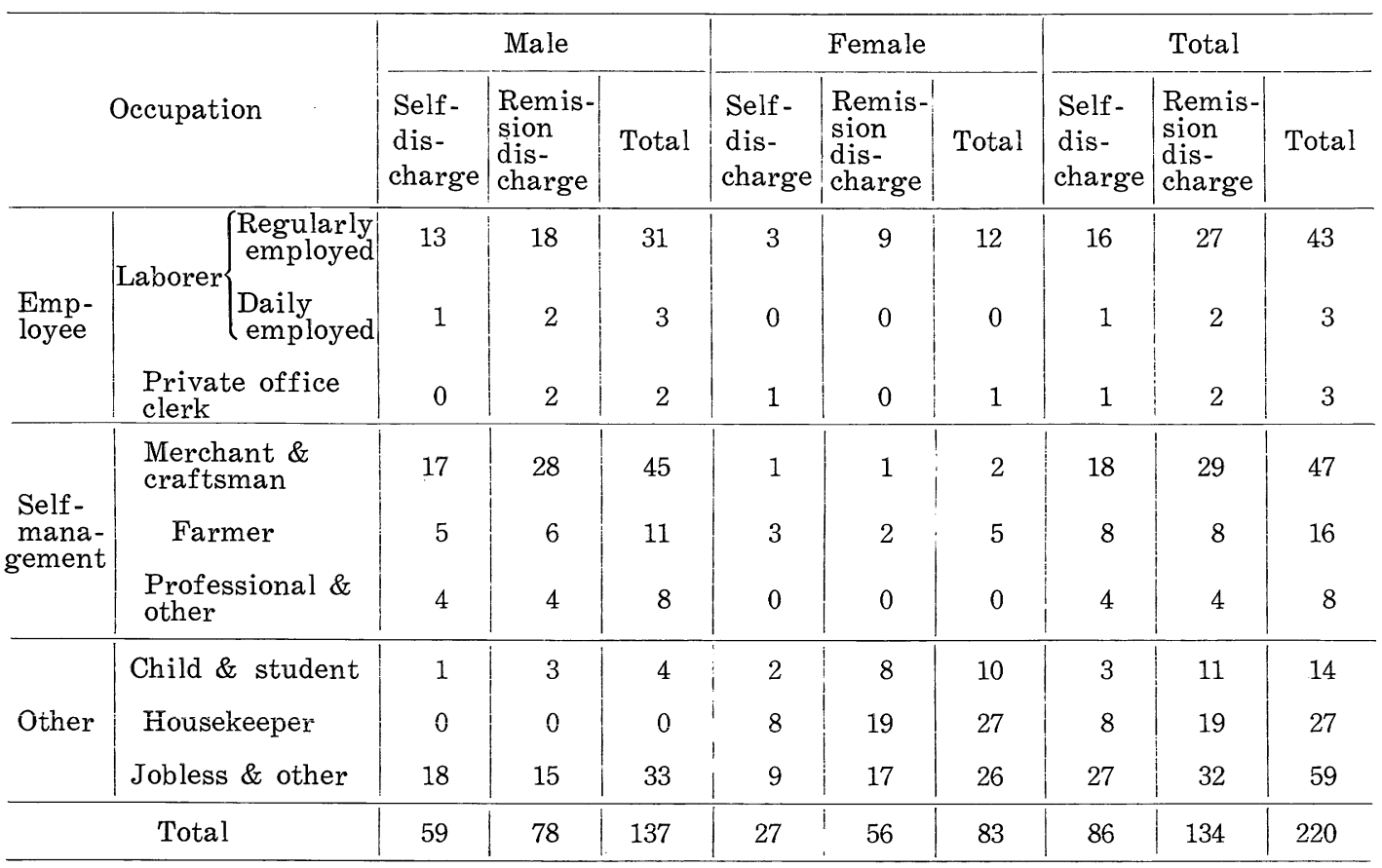

No significant differance

TABLE 4

By way of case discovery, 1969-1971, Kurume

\begin{tabular}{|c|c|c|c|c|c|c|c|c|c|}
\hline \multirow[b]{2}{*}{ Way of discovery } & \multicolumn{3}{|c|}{ Male } & \multicolumn{3}{|c|}{ Female } & \multicolumn{3}{|c|}{ Total } \\
\hline & $\begin{array}{l}\text { Self- } \\
\text { dis- } \\
\text { charge }\end{array}$ & $\begin{array}{l}\text { Remis- } \\
\text { sion } \\
\text { dis- } \\
\text { charge }\end{array}$ & Total & $\begin{array}{l}\text { Self- } \\
\text { dis- } \\
\text { charge }\end{array}$ & $\begin{array}{l}\text { Remis- } \\
\text { sion } \\
\text { dis- } \\
\text { charge }\end{array}$ & Total & $\begin{array}{l}\text { Self- } \\
\text { dis- } \\
\text { charge }\end{array}$ & $\begin{array}{l}\text { Remis- } \\
\text { sion } \\
\text { dis- } \\
\text { charge }\end{array}$ & Total \\
\hline Physical check-up & $\begin{array}{c}1 \\
(1.6)\end{array}$ & $\begin{array}{c}0 \\
(0)\end{array}$ & $\begin{array}{c}1 \\
(0.7)\end{array}$ & $\begin{array}{c}1 \\
(3.6)\end{array}$ & $\begin{array}{c}2 \\
(3.5)\end{array}$ & $\begin{array}{c}3 \\
(3.5)\end{array}$ & $\begin{array}{c}2 \\
(2.2)\end{array}$ & $\begin{array}{c}2 \\
(1.5)\end{array}$ & $\begin{array}{c}4 \\
(1.8)\end{array}$ \\
\hline Mass survey & $\begin{array}{c}15 \\
(24.2)\end{array}$ & $\begin{array}{c}7 \\
(8.8)\end{array}$ & $\begin{array}{c}22 \\
(15.5)\end{array}$ & $\begin{array}{c}3 \\
(10.7)\end{array}$ & $\begin{array}{c}10 \\
(17.5)\end{array}$ & $\begin{array}{c}13 \\
(15.3)\end{array}$ & $\begin{array}{c}18 \\
(20.0)\end{array}$ & $\begin{array}{c}17 \\
(12.4)\end{array}$ & $\begin{array}{c}35 \\
(15.4)\end{array}$ \\
\hline $\begin{array}{l}\text { On receiving treat- } \\
\text { ment at medical } \\
\text { facilities }\end{array}$ & $\begin{array}{c}46 \\
(74.2)\end{array}$ & $\begin{array}{c}73 \\
(91.2)\end{array}$ & $\begin{array}{c}119 \\
(83.8)\end{array}$ & $\begin{array}{c}24 \\
(85.7)\end{array}$ & $\begin{array}{c}45 \\
(79.0)\end{array}$ & $\begin{array}{c}69 \\
(81.2)\end{array}$ & $\begin{array}{c}70 \\
(77.8)\end{array}$ & $\begin{array}{c}118 \\
(86.1)\end{array}$ & $\begin{array}{l}188 \\
(82.8)\end{array}$ \\
\hline Total & 62 & 80 & 142 & 28 & 57 & 85 & 90 & 137 & 227 \\
\hline & & $\begin{array}{c}x^{2}=7.8 \\
n=2 \\
0.01<\mathrm{P}\end{array}$ & 0.02 & & $\begin{array}{c}x^{2}=0 . \\
\mathrm{n}=2 \\
0.7<\mathrm{P}<\end{array}$ & & & & \\
\hline
\end{tabular}


TABLE 5

Roentogenological finding on admission

(\%)

\begin{tabular}{|c|c|c|c|c|c|c|c|c|c|}
\hline \multirow[b]{2}{*}{$\begin{array}{l}\text { Roentogenological } \\
\text { finding }\end{array}$} & \multicolumn{3}{|c|}{ Male } & \multicolumn{3}{|c|}{ Female } & \multicolumn{3}{|c|}{ Total } \\
\hline & $\begin{array}{l}\text { Self- } \\
\text { dis- } \\
\text { charge }\end{array}$ & $\mid \begin{array}{l}\text { Remis }- \\
\text { sion } \\
\text { dis- } \\
\text { charge }\end{array}$ & Total & $\begin{array}{l}\text { Self- } \\
\text { dis- } \\
\text { charge }\end{array}$ & $\mid \begin{array}{l}\text { Remis- } \\
\text { sion } \\
\text { dis- } \\
\text { charge }\end{array}$ & Total & $\mid \begin{array}{l}\text { Self - } \\
\text { dis- } \\
\text { charge }\end{array}$ & \begin{tabular}{|l|} 
Remis- \\
sion \\
dis- \\
charge
\end{tabular} & Total \\
\hline Type I & $\begin{array}{c}7 \\
(11.3)\end{array}$ & $\begin{array}{c}2 \\
(2.5)\end{array}$ & $\begin{array}{c}9 \\
(6.3)\end{array}$ & $\begin{array}{c}3 \\
(10.7)\end{array}$ & $\begin{array}{c}2 \\
(3.5)\end{array}$ & $\begin{array}{c}5 \\
(5.9)\end{array}$ & $\begin{array}{c}10 \\
(11.1)\end{array}$ & $\begin{array}{c}4 \\
(2.9)\end{array}$ & $\begin{array}{c}14 \\
(6.2)\end{array}$ \\
\hline Type II & $\begin{array}{c}49 \\
(79.0)\end{array}$ & $\begin{array}{c}59 \\
(73.7)\end{array}$ & $\begin{array}{c}108 \\
(76.1)\end{array}$ & $\begin{array}{c}18 \\
(64.3)\end{array}$ & $\begin{array}{c}34 \\
(59.7)\end{array}$ & $\begin{array}{c}52 \\
(61.2)\end{array}$ & $\begin{array}{c}67 \\
(74.4) \\
\end{array}$ & $\begin{array}{c}93 \\
(67.9)\end{array}$ & $\begin{array}{c}160 \\
(70.5)\end{array}$ \\
\hline Type III & $\begin{array}{c}6 \\
(9.7)\end{array}$ & $\begin{array}{c}19 \\
(23.8)\end{array}$ & $\begin{array}{c}25 \\
(17.6)\end{array}$ & $\begin{array}{c}7 \\
(25.0)\end{array}$ & $\begin{array}{c}21 \\
(36.8)\end{array}$ & $\begin{array}{c}28 \\
(32.9)\end{array}$ & $\begin{array}{c}13 \\
(14.4)\end{array}$ & $\begin{array}{c}40 \\
(29.2)\end{array}$ & $\begin{array}{c}53 \\
(23.3)\end{array}$ \\
\hline Total & 62 & 80 & 142 & 28 & 57 & 85 & 90 & 137 & 227 \\
\hline & & $\begin{array}{c}x^{2}=8.31 \\
\mathrm{n}=2 \\
0.01<\mathrm{P}\end{array}$ & 02 & & $\begin{array}{c}x^{2}=2.52 \\
\mathrm{n}=2 \\
0.2<\mathrm{P}<\end{array}$ & & & & \\
\hline
\end{tabular}

TABLE 6

Bacteriological state on admission

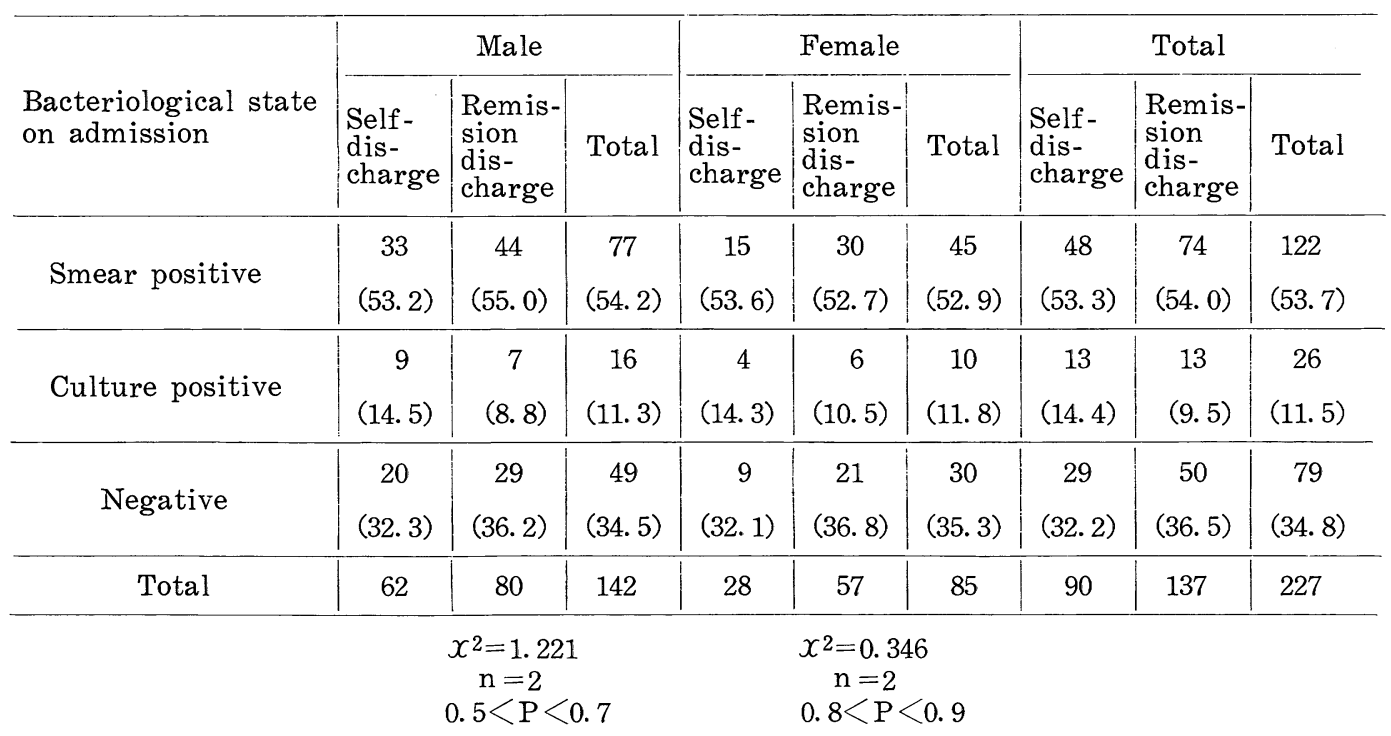


5) Bacteriological state on admission (Table 6)

The bacteriological state on admission was classified into negative, smear positive and culture positive. Of the total patients, $53.7 \%$ were smear positive, $11.5 \%$ were culture positive and $34.8 \%$ were negative. There was no significant difference between each group.

6) Number of changing doctors or hospitals (Table 7)

Of the total patients, $81.0 \%$ changed no doctors or hospitals, $15.0 \%$ changed once and $4.0 \%$ changed twice. There was no significant difference between male patients but more female patients who discharged by themselves changed dectors or hospitals with significant difference.

7) Level of education (Table 8)

The level of education was classified into compulsory education and middle school of old system or high school of new system. Of the total patients, 85.9
$\%$ received only compulsory education. Those discharged by themselves, both male and female patients, received less education significantly.

8) Patient's attitude toward treatment (Table 9)

The patient's attitude toward treatment was classified into very cooperative, general and uncooperative. Of the male patients discharged by themselves, $19.4 \%$ were very cooperative and $53.2 \%$ were uncooperative. Of the male patients discharged in favorable condition, $58.7 \%$ were very cooperative and only $8.8 \%$ were uncooperative. Among the female patients discharged by themselves, $21.4 \%$ were very cooperative and $42.9 \%$ were uncooperative. Among the female patients discharged in favorable condition, $77.2 \%$ were very cooperative and only $3.5 \%$ were uncooperative. Both male and female patients, those discharged in favorable condition were more cooperative than the self-discharged.

TABLE 7

Number of changing doctors or hospitals

(\%)

\begin{tabular}{|c|c|c|c|c|c|c|c|c|c|}
\hline \multirow[b]{2}{*}{$\begin{array}{l}\text { Changing dators } \\
\text { or hospitals }\end{array}$} & \multicolumn{3}{|c|}{ Male } & \multicolumn{3}{|c|}{ Female } & \multicolumn{3}{|c|}{ Total } \\
\hline & $\begin{array}{l}\text { Self- } \\
\text { dis- } \\
\text { charge }\end{array}$ & $\begin{array}{l}\text { Remis- } \\
\text { sion } \\
\text { dis- } \\
\text { charge }\end{array}$ & Total & $\begin{array}{l}\text { Self- } \\
\text { dis- } \\
\text { charge }\end{array}$ & $\begin{array}{l}\text { Remis- } \\
\text { sion } \\
\text { dis- } \\
\text { charge }\end{array}$ & Total & $\begin{array}{l}\text { Self- } \\
\text { dis- } \\
\text { charge }\end{array}$ & $\begin{array}{l}\text { Remis- } \\
\text { sion } \\
\text { dis- } \\
\text { charge }\end{array}$ & Total \\
\hline None & $\begin{array}{c}45 \\
(72.6)\end{array}$ & $\begin{array}{c}63 \\
(78.8)\end{array}$ & $\begin{array}{c}108 \\
(76.1)\end{array}$ & $\begin{array}{c}22 \\
(78.5)\end{array}$ & $\begin{array}{c}54 \\
(94.7)\end{array}$ & $\begin{array}{c}76 \\
(89.4)\end{array}$ & $\begin{array}{c}67 \\
(74.4)\end{array}$ & $\begin{array}{l}117 \\
(85.4)\end{array}$ & $\begin{array}{c}184 \\
(81.0)\end{array}$ \\
\hline Once & $\begin{array}{c}13 \\
(21.0)\end{array}$ & $\begin{array}{c}14 \\
(17.5)\end{array}$ & $\begin{array}{c}27 \\
(19.0)\end{array}$ & $\begin{array}{c}5 \\
(17.9)\end{array}$ & $\begin{array}{c}2 \\
(3.5)\end{array}$ & $\begin{array}{c}7 \\
(8.2)\end{array}$ & $\begin{array}{c}18 \\
(20.0)\end{array}$ & $\begin{array}{c}16 \\
(11.7)\end{array}$ & $\begin{array}{c}34 \\
(15.0)\end{array}$ \\
\hline Twice & $\begin{array}{c}4 \\
(6.4)\end{array}$ & $\begin{array}{c}3 \\
(3.7)\end{array}$ & $\begin{array}{c}7 \\
(4.9)\end{array}$ & $\begin{array}{c}1 \\
(3.6)\end{array}$ & $\begin{array}{c}1 \\
(1.8)\end{array}$ & $\begin{array}{c}2 \\
(2.4)\end{array}$ & $\begin{array}{c}5 \\
(5.6)\end{array}$ & $\begin{array}{c}4 \\
(2.9)\end{array}$ & $\begin{array}{c}9 \\
(4.0)\end{array}$ \\
\hline Total & 62 & 80 & 142 & 28 & 57 & 85 & 90 & 137 & 227 \\
\hline & & $\begin{array}{c}x^{2}=0.91 \\
n=2 \\
0.5<\mathrm{P}\end{array}$ & & & $\begin{array}{c}x^{2}=5.50 \\
n=2 \\
0.05<P\end{array}$ & 0.1 & & & \\
\hline
\end{tabular}


9) The income level (Table 10)

The income level of the patient's family was classified into 4 grades according to the tax the family paid. There was no significant difference between each group.

10) Duty of householde conomy (Table 11)

$62.3 \%$ of the male patients discharg- ed by themselves, $57.0 \%$ of the male patients discharged in favorable condition, $10.7 \%$ of the female patients discharged by themselves and $7.0 \%$ of the female patients discharged in favorable condition replied that they had duty of household economy. However there was no significant difference between each group.

TABLE 8

By, level of education

$(\%)$

\begin{tabular}{|c|c|c|c|c|c|c|c|c|c|}
\hline \multirow[b]{2}{*}{ Level of education } & \multicolumn{3}{|c|}{ Male } & \multicolumn{3}{|c|}{ Female } & \multicolumn{3}{|c|}{ Total - } \\
\hline & $\begin{array}{l}\text { Self- } \\
\text { dis- } \\
\text { charge }\end{array}$ & $\begin{array}{l}\text { Remis- } \\
\text { sion } \\
\text { dis- } \\
\text { charge }\end{array}$ & Total & $\begin{array}{l}\text { Self-! } \\
\text { dis- } \\
\text { charge }\end{array}$ & $\begin{array}{l}\text { Remis- } \\
\text { sion } \\
\text { dis- } \\
\text { charge }\end{array}$ & Total & $\begin{array}{l}\text { Self-c } \\
\text { dis- } \\
\text { charge }\end{array}$ & $\begin{array}{l}\text { Remis- } \\
\text { sion } \\
\text { dis- } \\
\text { charge }\end{array}$ & Total \\
\hline $\begin{array}{l}\text { Middle school of old } \\
\text { system or high school } \\
\text { of new sytem }\end{array}$ & $\begin{array}{c}1 \\
(2.0)\end{array}$ & $\begin{array}{c}10 \\
(14.1)\end{array}$ & $\begin{array}{l}11 \\
(9.1)\end{array}$ & $\begin{array}{c}2 \\
(9.1)\end{array}$ & $\begin{array}{c}14 . \\
(29.2)\end{array}$ & $\begin{array}{c}16 \\
(22.9)\end{array}$ & $\begin{array}{c}3 \\
(4.2)\end{array}$ & $\begin{array}{c}24 \\
(20.2)\end{array}$ & $\begin{array}{c}27 \\
(14.1)\end{array}$ \\
\hline $\begin{array}{l}\text { Compulsory } \\
\text { education }\end{array}$ & $\begin{array}{c}49 \\
(98.0)\end{array}$ & $\mid \begin{array}{c}61 \\
(85.9)\end{array}$ & $\begin{array}{c}110 \\
(90.9)\end{array}$ & $\begin{array}{c}20 \\
(90.9)\end{array}$ & $\begin{array}{c}34 \\
(70.8)\end{array}$ & $\begin{array}{c}54 \\
(77.1)\end{array}$ & $\begin{array}{c}69 \\
(95.8)\end{array}$ & $\begin{array}{c}95 \\
(79.8)\end{array}$ & $\begin{array}{l}164 \\
(85.9)\end{array}$ \\
\hline Total & 50 & 71 & 121 & 22 & 48 & 70 & 72 & 119 & 191 \\
\hline & & $\begin{array}{c}x^{2}=5.18 \\
n=1 \\
0.02<\mathrm{P}<\end{array}$ & 0.05 & $\therefore$ & $\begin{array}{c}x^{2}=3.44 \\
n=1 \\
0.05<P\end{array}$ & $<0.10$ & & & \\
\hline
\end{tabular}

TABLE 9

Patient's attitude toward treatment

\begin{tabular}{|c|c|c|c|c|c|c|c|c|c|}
\hline \multirow[b]{2}{*}{$\begin{array}{l}\text { Attitude toward } \\
\text { treatment }\end{array}$} & \multicolumn{3}{|c|}{ Male } & \multicolumn{3}{|c|}{ Female } & \multicolumn{3}{|c|}{ Total } \\
\hline & $\begin{array}{l}\text { Self- } \\
\text { dis- } \\
\text { charge }\end{array}$ & \begin{tabular}{|} 
Remis- \\
sion \\
dis- \\
charge
\end{tabular} \mid & Total & $\begin{array}{l}\text { Self- } \\
\text { dis- } \\
\text { charge }\end{array}$ & $\begin{array}{l}\text { Remis- } \\
\text { sion } \\
\text { dis- } \\
\text { charge }\end{array}$ & Total & $\begin{array}{l}\text { Self- } \\
\text { dis- } \\
\text { charge }\end{array}$ & $\begin{array}{l}\text { Remis- } \\
\text { sion } \\
\text { dis- } \\
\text { charge }\end{array}$ & Total \\
\hline Very cooperative & $\begin{array}{c}12 \\
(19.4)\end{array}$ & $\begin{array}{c}47 \\
(58.7)\end{array}$ & $\begin{array}{c}59 \\
(41.5)\end{array}$ & $\begin{array}{c}6 \\
(21.4)\end{array}$ & $\begin{array}{c}44 \\
(77.2)\end{array}$ & $\begin{array}{c}50 \\
(58.8)\end{array}$ & $\begin{array}{c}18 \\
(20.0)\end{array}$ & $\begin{array}{r}91 \\
(66.4)\end{array}$ & $\begin{array}{c}109 \\
(48.0)\end{array}$ \\
\hline General & $\begin{array}{c}17 \\
(27.4)\end{array}$ & $\begin{array}{c}26 \\
(32.5)\end{array}$ & $\begin{array}{c}43 \\
(30.3)\end{array}$ & $\begin{array}{c}10 \\
(35.7)\end{array}$ & $\begin{array}{c}11 \\
\text { (19. 3) }\end{array}$ & $\begin{array}{c}21 \\
(24.7)\end{array}$ & $\begin{array}{c}27 \\
(30.0)\end{array}$ & $\begin{array}{c}37 \\
(27.0)\end{array}$ & $\begin{array}{c}64 \\
(28.2)\end{array}$ \\
\hline Uncooperative & $\begin{array}{c}33 \\
(53.2)\end{array}$ & $\begin{array}{c}7 \\
(8.8)\end{array}$ & $\begin{array}{c}40 \\
(28.2)\end{array}$ & $\begin{array}{c}12 \\
(42.9)\end{array}$ & $\begin{array}{c}2 \\
(3.5)\end{array}$ & $\begin{array}{c}14 \\
(16.5)\end{array}$ & $\begin{array}{c}45 \\
(50.0)\end{array}$ & $\begin{array}{c}9 \\
(6.6)\end{array}$ & $\begin{array}{c}54 \\
(23.8)\end{array}$ \\
\hline Total & 62 & 80 & 142 & 28 & 57 & 85 & 90 & 137 & 227 \\
\hline & & $\begin{array}{c}x^{2}=37.8 \\
\mathrm{n}=2 \\
\mathrm{P}<0.001\end{array}$ & & & $\begin{array}{c}x^{2}=29 . \\
n=2 \\
\mathrm{P}<0.00\end{array}$ & & & & \\
\hline
\end{tabular}


TABLE 10

The income level

\begin{tabular}{|c|c|c|c|c|c|c|c|c|c|}
\hline \multirow[b]{2}{*}{ Income level } & \multicolumn{3}{|c|}{ Male } & \multicolumn{3}{|c|}{ Female } & \multicolumn{3}{|c|}{ Total } \\
\hline & $\begin{array}{l}\text { Self- } \\
\text { dis- } \\
\text { charge }\end{array}$ & $\begin{array}{l}\text { Remis- } \\
\text { sion } \\
\text { dis- } \\
\text { charge }\end{array}$ & Total & $\begin{array}{l}\text { Self- } \\
\text { dis- } \\
\text { charge }\end{array}$ & $\begin{array}{l}\text { Remis- } \\
\text { sion } \\
\text { dis- } \\
\text { charge }\end{array}$ & Total & $\begin{array}{l}\text { Self - } \\
\text { dis- } \\
\text { charge }\end{array}$ & $\begin{array}{l}\text { Remis- } \\
\text { sion } \\
\text { dis- } \\
\text { charge }\end{array}$ & Total \\
\hline Non-taxed $\quad\left(A_{1}\right)$ & $\begin{array}{c}19 \\
(30.6)\end{array}$ & $\begin{array}{c}29 \\
(36.3)\end{array}$ & $\begin{array}{c}48 \\
(33.8)\end{array}$ & $\begin{array}{c}6 \\
(21.4)\end{array}$ & $\begin{array}{c}15 \\
(26.3)\end{array}$ & $\begin{array}{c}21 \\
(24.7)\end{array}$ & $\begin{array}{c}25 \\
(27.8)\end{array}$ & $\begin{array}{c}44 \\
(32.1)\end{array}$ & $\begin{array}{c}69 \\
(30.4)\end{array}$ \\
\hline Limited taxed ( $\left.\mathrm{A}_{2}\right)$ & $\begin{array}{c}27 \\
(43.5)\end{array}$ & $\begin{array}{c}27 \\
(33.7)\end{array}$ & $\begin{array}{c}54 \\
(38.1)\end{array}$ & $\begin{array}{c}14 \\
(50.0)\end{array}$ & $\begin{array}{c}23 \\
(40.3)\end{array}$ & $\begin{array}{c}37 \\
(43.6)\end{array}$ & $\begin{array}{c}41 \\
(45.6)\end{array}$ & $\begin{array}{c}50 \\
(36.5)\end{array}$ & $\begin{array}{c}91 \\
(40.1)\end{array}$ \\
\hline $\begin{array}{l}\text { Income tax below } \\
6600 \text { yen }\left(B_{1}, B_{2}\right)\end{array}$ & $\begin{array}{c}13 \\
(21.0)\end{array}$ & $\begin{array}{c}18 \\
(22.5)\end{array}$ & $\begin{array}{c}31 \\
(21.8)\end{array}$ & $\begin{array}{c}4 \\
(14.3)\end{array}$ & $\begin{array}{c}12 \\
(21.1)\end{array}$ & $\begin{array}{c}16 \\
(18.8)\end{array}$ & $\begin{array}{c}17 \\
(18.9)\end{array}$ & $\begin{array}{c}30 \\
(21.9)\end{array}$ & $\begin{array}{c}47 \\
(20.7)\end{array}$ \\
\hline $\begin{array}{l}\text { Income tax over } \\
6600 \text { yen } \quad\left(B_{3}\right)\end{array}$ & $\begin{array}{c}3 \\
(4.9)\end{array}$ & $\begin{array}{c}6 \\
(7.5)\end{array}$ & $\begin{array}{c}9 \\
(6.3)\end{array}$ & $\begin{array}{c}4 \\
(14.3)\end{array}$ & $\begin{array}{c}7 \\
(12.3)\end{array}$ & $\begin{array}{c}11 \\
(12.9)\end{array}$ & $\begin{array}{c}7 \\
(7.8)\end{array}$ & $\begin{array}{c}13 \\
(9.5)\end{array}$ & $\begin{array}{l}20 \\
(8.8)\end{array}$ \\
\hline Total & 62 & 80 & 142 & 28 & 57 & 85 & 90 & 137 & 227 \\
\hline & & $\begin{array}{c}x^{2}=1.63 \\
n=3 \\
0.5<P<\end{array}$ & & & $\begin{array}{c}x^{2}=1.0 \\
n=3 \\
0.7<0.8\end{array}$ & & & & \\
\hline
\end{tabular}

TABLE 11

Duty of household economy

\begin{tabular}{|c|c|c|c|c|c|c|c|c|c|}
\hline \multirow[b]{2}{*}{$\begin{array}{l}\text { Duty of household } \\
\text { economy }\end{array}$} & \multicolumn{3}{|c|}{ Male } & \multicolumn{3}{|c|}{ Female } & \multicolumn{3}{|c|}{ Total } \\
\hline & $\begin{array}{l}\text { Self- } \\
\text { dis- } \\
\text { charge }\end{array}$ & $\left|\begin{array}{l}\text { Remis- } \\
\text { sion } \\
\text { dis- } \\
\text { charge }\end{array}\right|$ & Total & $\begin{array}{l}\text { Self- } \\
\text { dis- } \\
\text { charge }\end{array}$ & $\left|\begin{array}{l}\text { Remis- } \\
\text { sion } \\
\text { dis- } \\
\text { charge }\end{array}\right|$ & Total & $\begin{array}{l}\text { Self- } \\
\text { dis- } \\
\text { charge }\end{array}$ & $\begin{array}{l}\text { Remis- } \\
\text { sion } \\
\text { dis- } \\
\text { charge }\end{array}$ & Total \\
\hline Yes & $\begin{array}{c}41 \\
(62.3)\end{array}$ & $\begin{array}{c}45 \\
(57.0)\end{array}$ & $\begin{array}{c}86 \\
(61.4)\end{array}$ & $\begin{array}{c}3 \\
(10.7)\end{array}$ & $\begin{array}{c}4 \\
(7.0)\end{array}$ & $\begin{array}{c}7 \\
(8.2)\end{array}$ & $\begin{array}{c}44 \\
(49.4)\end{array}$ & $\begin{array}{c}49 \\
(36.0)\end{array}$ & $\begin{array}{c}93 \\
(41.3)\end{array}$ \\
\hline No & $\begin{array}{c}20 \\
(37.7)\end{array}$ & $\begin{array}{c}34 \\
(43.0)\end{array}$ & $\begin{array}{c}54 \\
(38.6)\end{array}$ & $\begin{array}{c}25 \\
(89.3)\end{array}$ & $\begin{array}{c}53 \\
(93.0)\end{array}$ & $\begin{array}{c}78 \\
(91.8)\end{array}$ & $\begin{array}{c}45 \\
(50.6)\end{array}$ & $\begin{array}{c}87 \\
(64.0)\end{array}$ & $\begin{array}{c}132 \\
(58.7)\end{array}$ \\
\hline Total & 61 & 79 & 140 & 28 & 57 & 85 & 89 & 136 & 225 \\
\hline \multicolumn{10}{|c|}{$\begin{array}{c}x^{2}=1.527 \\
\mathrm{n}=1 \\
0.2<\mathrm{P}<0.3\end{array}$} \\
\hline
\end{tabular}


11) Hands for housekeeping (Table 12)

If there is other person who can take over the housekeeping, the patient can be admitted to hospital without much worry, especially for female patients. Among the female patients, 60.7 $\%$ of the self-discharged and $21.4 \%$ of the remission discharged replied having no hands for housekeeping during the admission period, the difference was significant. But there was no significant difference between male patients. For female patients without hands for housekeeping, it would be difficult for them to be admitted to hospital for a long time.

TABLE 12

Hands for housekeeping

(\%)

\begin{tabular}{|c|c|c|c|c|c|c|c|c|c|}
\hline \multirow[b]{2}{*}{$\begin{array}{l}\text { Hands for house- } \\
\text { keeping }\end{array}$} & \multicolumn{3}{|c|}{ Male } & \multicolumn{3}{|c|}{ Female } & \multicolumn{3}{|c|}{ Total } \\
\hline & $\begin{array}{l}\text { Self- } \\
\text { dis- } \\
\text { charge }\end{array}$ & $\mid \begin{array}{l}\text { Remis- } \\
\text { sion } \\
\text { dis- } \\
\text { charge }\end{array}$ & Total & $\begin{array}{l}\text { Self- } \\
\text { dis- } \\
\text { charge }\end{array}$ & $\begin{array}{l}\text { Remis- } \\
\text { sion } \\
\text { dis- } \\
\text { charge }\end{array}$ & Total & $\begin{array}{l}\text { Self- } \\
\text { dis- } \\
\text { charge }\end{array}$ & $\begin{array}{l}\text { Remis- } \\
\text { sion } \\
\text { dis- } \\
\text { charge }\end{array}$ & Total \\
\hline Yes & $\begin{array}{c}53 \\
(91.4)\end{array}$ & $\begin{array}{c}70 \\
(90.9)\end{array}$ & $\begin{array}{c}123 \\
(90.4)\end{array}$ & $\begin{array}{c}11 \\
(39.3)\end{array}$ & $\begin{array}{c}44 \\
(78.6)\end{array}$ & $\begin{array}{c}55 \\
(65.5)\end{array}$ & $\begin{array}{c}64 \\
(73.6)\end{array}$ & $\begin{array}{c}114 \\
(85.7)\end{array}$ & $\begin{array}{c}178 \\
(80.9)\end{array}$ \\
\hline No & $\begin{array}{c}6 \\
(8.6)\end{array}$ & $\begin{array}{c}7 \\
(9.1)\end{array}$ & $\begin{array}{c}13 \\
(9.6)\end{array}$ & $\begin{array}{c}17 \\
(60.7)\end{array}$ & $\begin{array}{c}12 \\
(21.4)\end{array}$ & $\begin{array}{c}29 \\
(34.5)\end{array}$ & $\begin{array}{c}23 \\
(26.4)\end{array}$ & $\begin{array}{c}19 \\
(14.3)\end{array}$ & $\begin{array}{c}42 \\
(19.1)\end{array}$ \\
\hline Total & 59 & 77 & 136 & 28 & 56 & 84 & 87 & 133 & 220 \\
\hline & & $\begin{array}{c}x^{2}=0.04 \\
n=1 \\
0.8<\mathrm{P}<\end{array}$ & & & $\begin{array}{c}x^{2}=12 \\
n=1 \\
\mathrm{P}<0.01\end{array}$ & & & & \\
\hline
\end{tabular}

TABLE 13

Attitude of the patient's family

(\%)

\begin{tabular}{|c|c|c|c|c|c|c|c|c|c|}
\hline \multirow[b]{2}{*}{$\begin{array}{l}\text { Attitude of the } \\
\text { family }\end{array}$} & \multicolumn{3}{|c|}{ Male } & \multicolumn{3}{|c|}{ Female } & \multicolumn{3}{|c|}{ Total } \\
\hline & $\begin{array}{l}\text { Self- } \\
\text { dis- } \\
\text { charge }\end{array}$ & $\mid \begin{array}{l}\text { Remis- } \\
\text { sion } \\
\text { dis- } \\
\text { charge }\end{array}$ & Total & $\begin{array}{l}\text { Self- } \\
\text { dis- } \\
\text { charge }\end{array}$ & $\mid \begin{array}{l}\text { Remis- } \\
\text { sion } \\
\text { dis- } \\
\text { charge }\end{array}$ & Total & $\begin{array}{l}\text { Self- } \\
\text { dis- } \\
\text { charge }\end{array}$ & \begin{tabular}{|l|} 
Remis- \\
sion \\
dis- \\
charge
\end{tabular} & Total \\
\hline Good & $\begin{array}{c}19 \\
(31.7)\end{array}$ & $\begin{array}{c}36 \\
(46.7)\end{array}$ & $\begin{array}{c}55 \\
(40.1)\end{array}$ & $\begin{array}{c}6 \\
(21.4)\end{array}$ & $\begin{array}{c}34 \\
(60.7)\end{array}$ & $\begin{array}{c}40 \\
(47.6)\end{array}$ & $\begin{array}{c}25 \\
(28.4)\end{array}$ & $\begin{array}{c}70 \\
(52.6)\end{array}$ & $\begin{array}{c}95 \\
(43.0)\end{array}$ \\
\hline General & $\begin{array}{c}25 \\
(41.6)\end{array}$ & $\begin{array}{c}32 \\
(41.6)\end{array}$ & $\begin{array}{c}57 \\
(41.6)\end{array}$ & $\begin{array}{c}16 \\
(57.2)\end{array}$ & $\begin{array}{c}18 \\
(32.2)\end{array}$ & $\begin{array}{c}34 \\
(40.5)\end{array}$ & $\begin{array}{c}41 \\
(46.6)\end{array}$ & $\begin{array}{c}50 \\
(37.6)\end{array}$ & $\begin{array}{c}91 \\
(41.2)\end{array}$ \\
\hline Bad & $\begin{array}{c}16 \\
(26.7)\end{array}$ & $\begin{array}{c}9 \\
(11.7)\end{array}$ & $\begin{array}{c}25 \\
(18.3)\end{array}$ & $\begin{array}{c}6 \\
(21.4)\end{array}$ & $\begin{array}{c}4 \\
(7.1)\end{array}$ & $\begin{array}{c}10 \\
(11.9)\end{array}$ & $\begin{array}{c}22 \\
(25.0)\end{array}$ & $\begin{array}{c}13 \\
(9.8)\end{array}$ & $\begin{array}{c}35 \\
(15.8)\end{array}$ \\
\hline Total & 60 & 77 & 137 & 28 & 56 & 84 & 88 & 133 & 221 \\
\hline & & $\begin{array}{c}x^{2}=6.05 \\
n=2 \\
0.02<P\end{array}$ & 0.05 & & $\begin{array}{c}x^{2}=12 . \\
n=2 \\
P<0.01\end{array}$ & & & & \\
\hline
\end{tabular}


12) The attitude of the patient's family (Table 13)

The attitude of the family also plays a role in the effect of treatment of tuberculosis. As a chronic disease, it may take several years to cure. Whether the family would pay warm attention to the patient for such a long time can influence the effect of treatment. 26.7 $\%$ of the families of the male patients discharged by themselves, $11.7 \%$ of the families of the male patients discharged in favorable condition, $21.4 \%$ of the families of the female patients discharged by themselves and $7.1 \%$ of the families of the female patients discharged in favorable condition paid little attention to the patients. The difference was significant between the self-discharged and the remission discharged.

\section{DISCUSSION}

The public expenditure system for treatment of tuberculosis has been put into effect since October 1, 1951. And after the revision of the items on May 1,1963 , it has become more effective in the spread of proper treatment for tuberculous patients. However there were still many patients discontinued to receive a more complete treatment even they need not to pay the medical fee. The author noted that there were many patients discharged by themselves for personal reasons before getting favorable improvement. So the author tried to analyse the chief factors of self-discharge and chose the patients discharged in favorable condition as controls.

12 chief factors concerning to host, agent and environment were investigated. They are the period of admission, occupation, way of case discovery, roentogenological finding on admission, bacteriological state on admission, num- ber of changing doctors or hospitals, level of education, patient's attitude toward treatment, income level, duty of household economy, hands for housekeeping and the attitude of the patient's family.

The factors without significant difference between the 2 groups included the period of admission, occupation, bacteriological state on admission, number of changing doctors or hospitals, the income level and the duty of household economy. It is interesting that there was no significant relationship between economic factor and self-discharge.

Those factors with significant difference were as follows. As to the roentogenological finding on admission, the male patients discharged by themselves were more of Type I and Type II and less of Type III. The level of education of the self-discharged was lower than that of those discharged in favorable condition for both male and female patients, 'which showed that education could influence on the treatment of tuberculosis. The more education one receives, the more one can understand the need of treating the disease. More of the self-discharged were uncooperative toward treatment, while most of those discharged in favorable condition were cooperative. For female patient, if there is no other person can take over the housekeeping, she may be too anixious to be admitted to hospital for a long time. In fact, many female patients discharged before getting favorable improvement for lack of hands for housekeeping. It is also necessary to have warm attention of the family in the course of long-term treatment. More of the families of the self-discharged paid less attention than those of the remission discharged. From the above data, we can see that the patient's own awareness of the disease, 
his cooperativeness toward treatment and warm attention of the family were indispensable to complete a long-term treatment. Economic factor did not play the whole role.

\section{ACKNOWLEDGEMENTS}

The author thanks Dr. Yoshio Kabashima, Director of Kurume Health Center, for his kindness.

\section{REFERENCES}

1) EsAKI, H.: Studies on the cause of death of the registered TB-patients: with spe- cial reference to the relation between pulmonary tuberculosis and the lung cancer. Kurume M. J., 17, 55-60, 1970.

2) ESAKI, H.: Epidemiological survey on the registered TB-patients in Kurume City. Human Ecol. \& Race Hyg, 37, 126, 1971.

3 ) EsAKI, H. and TAKeuchI, T. : Epidemiological studies on tuberculosis in Kurume City (Report I) A trend of the registered TBpatients. J. Kurume M. A., 35, 1611-1616, 1972.

4) EsAKI, H.: Epidemiological survey on the new cases of tuberculosis in Fukuoka Prefecture - especially on the actual condition within the jurisdiction of Kurume Health Center. Achiev. Reports of Chest Disease Research Council of Fukuoka Prefecture., 15-21, 1972. 